\title{
Médiévales
}

Langues, Textes, Histoire

\section{Manuscrits d'antidotaires médiévaux : quelques exemples du fonds latin de la Bibliothèque nationale de France}

Mireille Ausécache

\author{
(2) OpenEdition \\ Journals \\ Édition électronique \\ URL : https://journals.openedition.org/medievales/2283 \\ DOI : $10.4000 /$ medievales. 2283 \\ ISSN : $1777-5892$ \\ Éditeur \\ Presses universitaires de Vincennes \\ Édition imprimée \\ Date de publication : 1 juin 2007 \\ Pagination : 55-74 \\ ISBN : 978-2-84292-202-3 \\ ISSN : 0751-2708 \\ Référence électronique \\ Mireille Ausécache, "Manuscrits d'antidotaires médiévaux : quelques exemples du fonds latin de la \\ Bibliothèque nationale de France », Médiévales [En ligne], 52 I printemps 2007, mis en ligne le 06 \\ septembre 2009, consulté le 22 avril 2022. URL : http://journals.openedition.org/medievales/2283 ; \\ DOI : https://doi.org/10.4000/medievales.2283
}




\section{Mireille AUSÉCACHE}

\section{MANUSCRITS D'ANTIDOTAIRES MÉDIÉVAUX : quelques exemples du fonds latin de la Bibliothèque nationale de France}

Les antidotaires sont des recueils présentant des médicaments composés, préparations résultant de savants dosages de substances végétales surtout, mais aussi animales et minérales qui, lorsqu'elles sont utilisées seules, sont qualifiées de simples ${ }^{1}$. Ces ouvrages comportent des prescriptions et des recettes plus ou moins précises de préparations. Plus longs que les réceptaires ou recueils de recettes, ils sont en général organisés (classement par type de médicaments, onguents, sirops, électuaires... ou ordre alphabétique) et tendent vers un objectif plus ambitieux en rassemblant une sorte d'inventaire des médicaments composés susceptibles d'être prescrits par les médecins ${ }^{2}$.

1. La nécessité des médicaments composés est présentée ainsi dans le Livre des simples médecines : «Il y a plusieurs cas pour lesquels on inventa des médecines composées, cas où les médecines simples ne suffisaient pas, à savoir: la violence de la maladie, les maladies contraires ou opposées, les états contraires des membres ou organes du corps, la noblesse de ces derniers, la violence de la médecine ». Cf. Platearius, Le Livre des simples médecines, d'après le manuscrit français 12322 de la Bibliothèque nationale de Paris, traduction et adaptation de G. Malandin, Paris, 1990, p. 8.

2. À la fin du XII ${ }^{\mathrm{e}}$ siècle le médecin français Gilles de Corbeil ne tarit pas d'éloges sur Salerne, la ville où il fit ses études de médecine. Dans le prologue en prose de son traité Des louanges et vertus des médicaments composés, il évoque avec emphase l'Antidotaire de Salerne qui constitue d'après lui l'arme essentielle dont dispose le médecin pour lutter contre la maladie. Cet ouvrage présentant toutes les formes de médicaments composés utilisables, leurs prescriptions, leurs dosages et modes d'administration, il estime même qu'il est le principal guide du médecin débutant: "Qui ideo antidotarius inscribitur, quia in eo summa omnium antidotorum et medicaminum ad morborum curationes pertinentium, summatim et breuiter texitur. Priori secundum ordinem alphabeti omnium antidotorum, catarticorum, electuariorum, emplaustrorum, cathaplasmatum et unguentorum et ne discurram per singula, omnium pericie medicinali suffragantium uirtutes et potentie distinguntur et contra quas ualeant egritudines exprimitur et modi exibitionum determinantur. In eo singulorum medicaminum receptiones declarantur et omnium que recipiuntur in medicinis ponderum quantitates appenduntur ». $C f$. M. AusÉCACHE (éd.), Le «De uirtutibus et laudibus compositorum medicaminum» de Gilles de Corbeil (XII ${ }^{e}$ s.). Édition et commentaire, thèse de doctorat de l'EPHE, 2003, p. 130, à paraître ; L. Choulant (éd.), Aegidii Corboliensis carmina medica, Leipzig, 1826 ; C. VIEILLARD, Essai sur la société médicale 
Ils constituent avec les recueils consacrés aux simples ${ }^{3}$ et les diverses practice rédigées par les maîtres ${ }^{4}$ le fonds indispensable au médecin médiéval qui pendant longtemps réalise lui-même les préparations avant que cette tâche ne soit réservée aux apothicaires ${ }^{5}$. Ils sont pour l'historien de la médecine un témoignage essentiel sur les pratiques thérapeutiques et les théories qui les sous-tendent.

et religieuse au XII ${ }^{e}$ siècle. Gilles de Corbeil, médecin de Philippe-Auguste et chanoine de Notre Dame (1140-1224), Paris, 1908. Sur Salerne consulter les travaux de P. O. KRISTELLER et notamment «The school of Salerno : its development and its contribution to the history of learning », Studies in Renaissance thought and letters, vol. I, Rome, 1956, p. 495-551 ; Studi sulla Scuola medica salernitana, Naples, 1986.

3. La materia medica de Dioscoride traduite en latin circule en Occident à partir du $\mathrm{VI}^{\mathrm{e}}$ siècle. Cf. A. TouwAIDE, «Stratégies thérapeutiques : les médicaments », dans M. D. GrmeK (dir.), Histoire de la pensée médicale en Occident. 1. Antiquité et Moyen Âge, Paris, 1995, p. 227-237, sp. p. 236-237. L'ouvrage qui s'imposa ensuite est le De simplici medicina ou Circa instans, rédigé à Salerne au XII ${ }^{\mathrm{e}}$ siècle vraisemblablement par Matheus Platearius, traité qui connut un grand et durable succès : s'enrichissant de nouvelles rubriques, il fut rapidement traduit en langue vernaculaire et imprimé dès la fin du $\mathrm{Xv}^{\mathrm{e}}$ siècle. $C f$. H. Wölfel, Das Arzneidrogenbuch "Circa instans» in einer Fassung des XIII. Jahrhunderts aus der Universitäts Bibliothek Erlangen. Text und Kommentar als Beitrag zur Pflanzen und Drogenkunde des Mittelallters, Diss. Berlin, 1939 ;PlateArius, Le Livre des simples médecines, op. cit.

4. Il s'agit de manuels exposant les différentes maladies selon un ordre a capite ad calcem, précisant souvent leurs « causes », leurs «signes ». Les médicaments cités dans ce type de traités sont rapidement évoqués, la practica proposant fréquemment plusieurs méthodes thérapeutiques pour la même maladie. Nombre de ces ouvrages furent produits dans le cadre de l' «École de Salerne » : le De aegritudinum curatione tractatus, en particulier, se présente comme une sorte de compilation des practice de plusieurs maîtres salernitains. Cf. S. DE RENZI, Collectio salernitana, t. II, Naples, 1853, p. 81-385. Salerne n'est sans doute pas une «École» au sens strict mais plutôt un regroupement, dans le même lieu, de maîtres illustres et d'élèves sans lien institutionnel formel. Cf. P. O. KRISTELler, Studi sulla Scuola salernitana, op. cit., p. 56-57. Les maîtres salernitains sont les auteurs de nombreux traités et commentaires portant notamment sur des ouvrages de l'Antiquité redécouverts grâce au mouvement des traductions de l'arabe et du grec. Cf. D. JACQUART, « Aristotelian Thought in Salerno » dans P. Dronke (éd.), A History of Twelfth Century Western Philosophy, Cambridge, 1988, p. 407-428 ; D. JACQUART, F. MicheAU, La médecine arabe et l'occident médiéval, Paris, 1990, p. 87-129; P. O. KRISTELLER, « Bartholomaeus, Musandinus, Maurus of Salerno and other early commentators of the Articella with a tentative list of texts and manuscripts ", Studies in Renaissance thougt and letters, vol. III, Rome, 1993, p. 403-429. L'éclat de Salerne, particulièrement vif au XII ${ }^{\mathrm{e}}$ siècle, pâlit au XIII ${ }^{\mathrm{e}}$ siècle au profit de Montpellier et Bologne.

5. À partir du XII ${ }^{\mathrm{e}}$ siècle, à Salerne surtout, la profession médicale s'affirme non plus seulement en tant qu'ars mais en tant que physica s'appuyant sur de solides bases théoriques afin de garantir la qualité du diagnostic et de la prescription proposée. Cf. D. JACQUART, «Theorica et practica dans l'enseignement de la médecine à Salerne au XII ${ }^{\mathrm{e}}$ siècle », Vocabulaire des écoles et des méthodes d'enseignement au Moyen Âge, éd. O. WeIJERs, Turnhout, 1992, p. 102-110 ; repris dans D. JACQUART, La Science médicale occidentale entre deux renaissances, Aldershot Variorum, 1997, VII. Nombre de praticiens continuent de manier eux-mêmes le pilon ou de diriger les opérations de préparation des médicaments : des miniatures illustrent parfois ce thème, cf. L. MacKinney, Medical Illustrations in Medieval Manuscripts, Londres, 1965, p. 219, fig. 22. Cependant, peu à peu, le médecin devient essentiellement le prescripteur de médicaments préparés par d'autres. Parallèlement à la spécialisation du physicus, la profession d'apothicaire se structure, s'organise «sur fond de polémique et de réglementation » tant ce métier est la cible des critiques des médecins. Ainsi Gilles de Corbeil accuse-t-il, en des termes très violents, les 
Les antidotaires latins qui nous sont parvenus révèlent l'évolution de la pharmacopée au cours du Moyen Âge, évolution liée notamment à l'introduction de plantes et de procédés nouveaux dans la composition des recettes mais aussi aux différences de conception et d'organisation des ouvrages selon les périodes et les lieux de production. Sur le terrain de la pharmacologie comme sur celui des théories médicales, un tournant important se produit à Salerne aux $\mathrm{XI}^{\mathrm{e}}$-XII ${ }^{\mathrm{e}}$ siècles, moment marqué notamment par l'introduction de la pharmacologie arabe. L'histoire des antidotaires, comme celle de la pensée médicale, établit donc la distinction entre période «pré-salernitaine » et période « salernitaine ».

L'étude des antidotaires médiévaux est indissociable de celle de leurs manuscrits ${ }^{6}$, mais elle-même a pour préalable indispensable un travail de repérage et d'identification qui s'avère souvent difficile du fait des imprécisions des catalogues de bibliothèques et du nombre restreint de répertoires consacrés aux manuscrits médicaux ${ }^{7}$. Le problème majeur consiste alors à repérer et identifier ces textes parfois insérés anonymement au milieu d'autres traités. Le présent travail entend simplement proposer une sorte de parcours parmi quelques-uns des nombreux manuscrits médicaux médiévaux que recèle le fonds

«pharmacopoles, marchands d'espèces et d'aromates, ces fabricants de fards », d'incompétence, d'avarice, de fraude et tromperie mettant en péril les malades. Cf. J.-P. BÉNÉzET, Pharmacie et médicament en Méditerranée occidentale (XIII -XVI ${ }^{e}$ s.), Paris, 1999, p. 19 ; L. MoulinIER-BrogI, «Médecins et apothicaires dans l'Italie médiévale. Quelques aspects de leurs relations », dans Pharmacopoles et apothicaires. Les "pharmaciens » de l'Antiquité au Grand Siècle, études réunies par F. Collard et E. Samama, Paris, 2006 p. 119-134.

6. Les antidotaires restent, dans leur grande majorité, inédits. Le seul à avoir connu un réel succès d'édition est l'Antidotarium Nicolai : abondamment copié, commenté, traduit, il fut imprimé dès 1471. Cf. D. Goltz, Mittelalterliche Pharmazie und Medizin, Dargestellt an Geschichte und Inhalt des «Antidotarium Nicolai » mit einem Nachdruck der Druckfassung von 1471, Stuttgart, 1976 ; P. Dorveaux, Deux traductions françaises de l'Antidotarium de Nicolas de Salerne (XIV'$X V^{e}$ s.), Paris, 1896. De plus, le nombre de témoins conservés, la présentation des textes dans les manuscrits (annotations, gloses...), le contexte des recueils qui les conservent sont autant d'indices permettant d'appréhender la diffusion de ces ouvrages et le public auquel ils étaient destinés.

7. L'ouvrage de base reste L. ThorndiKe, P. KiBre, A Catalogue of Incipits of medieval scientific writings in latin, Londres, 1963 ; P. KIBRE, «Further Addenda et Corrigenda », Speculum, 43, 1968, p. 78-114. Citons également à titre d'exemples de répertoires spécialisés : G. BEAuJouAn, «Index des manuscrits médicaux du Moyen Âge conservés en Espagne », Mélanges de la Casa Velasquez, VIII, 1972, p. 161-221 repris dans G. Beaujouan, Science médiévale d'Espagne et d'alentour, Variorum, Aldershot, 1992, V. ; R. J. DuRLING, «Bibliographical Studies. A Guide to the Medical Manuscripts mentioned in Kristeller's Iter italicum », Traditio, 41, 1985, p. 341 365 - 44, 1988, p. 485-536 ; P. PANSIER, « Catalogue des manuscrits médicaux des Bibliothèques de France. Manuscrits latins des bibliothèques autres que la Bibliothèque nationale de Paris », Archiv für Geschichte der Medizin, 2, 1909, p. 1-46; L. SchubA, Die medizinischen Handschriften der "Codices Palatini latini » in der Vatikanischen Bibliothek-Katalog der Universitätsbibliothek Heidelberg, Band I, Wiesbaden, 1981... Pour la période «pré-salernitaine » $f$. H. E. Sigerist, «Studien und Texte zur frühmittelalterlichen Rezeptliteratur», Studien zur Geschichte der Medizin, 13, 1923, p. 187-195; A. BECCARIA, I codici di Medicina del Periodo Presalernitano (Secoli IX, X, XI), Rome, 1956 ; E. WICKERSHEIMER, Manuscrits latins de médecine du haut Moyen Âge dans les bibliothèques de France, Paris, 1966. 
latin de la Bibliothèque nationale de France ${ }^{8}$, afin de présenter quelques antidotaires représentatifs de l'évolution de cette littérature spécialisée .

Pour mesurer l'ampleur du changement qui s'opère au tournant des $\mathrm{XI}^{\mathrm{e}}-$ $\mathrm{XII}^{\mathrm{e}}$ siècles en matière de littérature pharmacologique, il convient de rappeler quelques caractéristiques des recueils antérieurs. Ouvrage célèbre, le De medicamentis de Marcellus de Bordeaux dit Empiricus ( $\mathrm{V}^{\mathrm{e}} \mathrm{s}$.) se trouve notamment dans le manuscrit lat. $6880^{10}$. Le catalogue ${ }^{11}$ donne comme titre: Marcelli Empirici Theodosii magni Archiatri liber medicamentorum ad filios et indique la présence de notes marginales. Cet ouvrage comporte des recettes de médicaments prescrits pour des maladies classées de la tête aux pieds en 36 chapitres. L'antidotaire est accompagné de lettres attribuées à Hippocrate, Pline, Celse, Vindicianus... et de précisions sur les poids et mesures des ingrédients, l'auteur amassant «tout ce qui lui paraît devoir être utile aux populations, trop souvent privées de médecins, en souhaitant qu'elles puissent s'aider elles-mêmes » ${ }^{12}$. C'est donc un ensemble considéré comme un tout. D'autres recueils présentent simplement des séries de recettes, de taille très inégale, les préparations étant classées par type de médicament ou bien décrites les unes après les autres sans ordre. Le manuscrit lat. $7028^{13}$ comporte à la suite de textes d'Isidore de Séville, Vindicianus et Celse des compositiones oleorum $\left(\mathrm{f}^{\mathrm{o}} 109 \mathrm{v}^{\circ}-111 \mathrm{v}^{0}\right)$, des préparations d'emplâtres ( $\left.\mathrm{f}^{\circ} 136\right)$, une benedictio confectionis medicinalis $\left(\mathrm{f}^{\circ} 136 \mathrm{v}^{\circ}\right)$. Suivent ensuite 9 textes médicaux puis, à la fin du recueil, de nouvelles recettes d'emplâtres (fo $\left.180-183 v^{\circ}\right)$ et d'électuaires $\left(f^{\circ} 184-191 v^{\circ}\right)$. Il s'agit là de réceptaires et non d'antidotaires tels que nous les avons définis. Très fréquemment, les manuscrits présentent des recettes éparpillées ou regroupées en courtes séries dans les marges ou les espaces laissés vides au milieu d'autres ouvrages et il n'est pas rare de trouver de tels ajouts dans un contexte littéraire ou théologique. C'est, par exemple, le cas du manuscrit BnF lat. $2849 \mathrm{~A}^{14}$ : le catalogue de la Bibliothèque royale ${ }^{15}$ indique que, entre deux traités d'Alcuin (De fide Sanctae Trinitatis ad Carolum magnum et De Trinitate questiones ad Fredegisum), sont copiés des anonymi fragmenta de remediis morborum $\left(\mathrm{f}^{\mathrm{o}} 18 \mathrm{v}^{0}-23 \mathrm{v}^{\circ}\right)$. Le texte commence ainsi : Incipit medicinalis scarsus de probatis causis. In primis ad caput doloris...

8. Je remercie J. Chandelier, L. Moulinier-Brogi et M. Nicoud pour leurs précieuses informations, fruit de leurs travaux parus depuis, sous forme d'un «Index des manuscrits médicaux latins conservés à la $\mathrm{BnF}$ », dans les Archives doctrinales et littéraires du Moyen Âge, $\mathrm{n}^{\circ} 73,2006$, p. 63-163.

9. Le choix a porté sur 4 antidotaires datables vraisemblablement des $\mathrm{XII}^{\mathrm{e}}$ et $\mathrm{XIII}{ }^{\mathrm{e}}$ siècles : l'Antidotarius magnus de Salerne et le Liber iste qui en est issu, le célèbre Antidotarium Nicolai et le Pomum ambre très différent des précédents. Une présentation rapide des manuscrits est donnée en annexe.

10. IX $^{\mathrm{e}}$ siècle ; $C f$. A. BeCCARIA, p. 145-147; E. WiCKeRsheImer, p. 70-72. Notice dans La Médecine médiévale à travers les manuscrits de la Bibliothèque Nationale, Paris, 1982, 60, p. 74.

11. Catalogus codicum manuscriptorum Bibliothecae Regiae, IV, Paris, 1744, p. 289.

12. D. Gourevitch, «Les voies de la connaissance : la médecine dans le monde romain », dans M. D. Grmek (dir.), Histoire de la pensée médicale en Occident. 1. Antiquité et Moyen Âge, Paris, 1995 , p. 95-122, sp. p. 121.

13. $\mathrm{XI}^{\mathrm{e}}$ siècle ; $C f$. A. BeCCARIA, p. 152-56 ; E. WiCKERSHEIMER, p. 80-86.

14. IX ${ }^{\mathrm{e}}$ siècle. $C f$. A. BeCCARIA, p. 140 ; E. WiCKERSHEIMER, p. 59-61.

15. Catalogus codicum manuscriptorum Bibliothecae Regiae, III, Paris, 1744 p. 341-342. 
Les exemples sont nombreux de ces éléments rapportés, ces mentions étant, du fait de leur éparpillement, le plus souvent ignorées des catalogues. Les médicaments, présentés ainsi, occupent alors une place subalterne dans les recueils. C'est à partir du $\mathrm{XI}^{\mathrm{e}}$ et surtout du $\mathrm{XII}^{\mathrm{e}}$ siècle que les choses changent, principalement sous l'impulsion des auteurs salernitains.

\section{L'Antidotarius magnus}

La «période salernitaine » est surtout marquée, dans le domaine de la pharmacologie, par la création de l'Antidotarius magnus ${ }^{16}$ alphabétique, daté vraisemblablement du tournant des $\mathrm{XI}^{\mathrm{e}}-\mathrm{XII}^{\mathrm{e}}$ siècles et qui reste à ce jour anonyme ${ }^{17}$. Outre le classement alphabétique ${ }^{18}$, il diffère également de ses prédécesseurs par la quantité des formules présentées. Cette réalisation marque réellement un tournant et ouvre ce que l'on pourrait appeler «l'Âge d'or » des antidotaires, période où ce type d'ouvrage acquiert ses lettres de noblesse, prend une place importante et donne lieu à des productions de manuscrits soignés et même luxueux.

Les manuscrits de l'Antidotarius magnus ont été recensés en 1999 par Andreas Kramer et Korinna Scheidt ${ }^{19}$ qui annonçaient également un projet d'édition critique. Leur liste comporte 13 manuscrits présentant un nombre fort variable de préparations : l'antidotaire de Florence ${ }^{20}$ qui serait, selon ces

16. Cette dénomination est la plus courante mais on peut trouver également l'appellation d'Antidotarium universale. Cf. le ms. Venise, Biblioteca Marciana, lat. 321 (XIV ${ }^{\mathrm{e}}$ s.), $\mathrm{f}^{\mathrm{o}}$ 166$179 \mathrm{v}^{\circ}$ : Glossulae exponentes antidotarium uniuersale. Inc. Liber iste quem in praesentiarum legendum assumpsimus ex multorum antidotis est compilatus... Il s'agit du Liber iste, ensemble de gloses de Platearius au Grand Antidotaire, ouvrage dont il sera question plus loin.

17. Il est parfois attribué à Constantin l'Africain, moine du Mont Cassin né en Afrique du Nord, mort vers 1087 et auteur de nombreuses traductions de l'arabe au latin, notamment celle de l'encyclopédie d'Ali ibn al-Abbas al-Magusi, connue en Occident sous le nom de Pantegni. Cet ouvrage se compose de 2 parties: une theorica de 10 livres et une practica également divisée en 10 livres. Le dixième livre de la practica est un antidotaire présentant des recettes sans ordre apparent mais il ne figure pas dans tous les témoins conservés, la practica étant souvent incomplète. $C f$. D. JACQUART, F. Micheau, La Médecine arabe et l'occident médiéval, Paris, 1990, p. 96-107 ; C. Burnett, D. JaCQuart (éds.), Constantine the African and 'Ali Ibnal-Abbas al-Magusi': The Pantegni and related texts, Leyde-New York-Cologne, 1994.

18. Ce classement porte sur la première lettre du nom du médicament. Nombre de préparations portent encore une dénomination vague, comme antidotum ou electuarium ad dolorem capitis mais on relève également des appellations précises dérivées du nom du principal composant ou de l'inventeur présumé : diaprunis, antidotum Adrianum, Pigra Galieni... En ce qui concerne l'Occident médiéval, le premier ouvrage organisé selon l'ordre alphabétique semble être le Librum de orthographia de Bède le Vénérable (VIII ${ }^{\mathrm{e}} \mathrm{s}$ ) et dans le domaine botanique, la traduction latine des 5 livres de la Materia medica de Dioscoride réalisée au $\mathrm{VI}^{\mathrm{e}}$ siècle (le «Dioscoride Lombard»), fut réorganisée au Xie dans l'ordre alphabétique. Cf. R. CHARTIERH. J. Martin (dir.), Histoire de l'édition française. T 1, Le livre conquérant, du Moyen Âge au milieu du XVII ${ }^{e}$ siècle, Paris, 1989.

19. A. Kramer, K. ScheidT, «Die Handschriften des Antidotarius magnus », Sudhoffs Archiv, 83, 1999, p. 109-116.

20. Firenze, Biblioteca Nazionale Centrale, Cod. Palat. Lat. 747. 
auteurs, très proche de l'archétype, contient 521 préparations ; le manuscrit de Bâle ${ }^{21}$ comporte plus de 1100 formules ; le manuscrit BnF lat. 16187 en comporte quant à lui 1239 ! D'après la liste établie par A. Kramer et K. Scheidt, la BnF possède trois manuscrits du Grand Antidotaire : les lat. 7009, 7010, 16187.

Le manuscrit lat. 7009 (première moitié du XIII ${ }^{\mathrm{e}}$ s.) présente 928 médicaments en 84 folios ${ }^{22}$. Le catalogue le présente comme un antidotaire alphabétique anonyme : anonymi antidotarium ordine alphabetico dispositum ${ }^{23}$. Il s'agit d'un manuscrit soigné dans lequel l'ordre alphabétique est mis en valeur par une initiale ornée à chaque changement de lettre. La nomenclature commence avec l'Aurea alexandrina et se termine au folio $84 \mathrm{v}^{\circ}$ avec la Zegzenia minor suivie de la mention explicit liber. D'autres recettes, de la même main mais ne s'inscrivant plus dans l'ordre alphabétique, complètent la page.

L'antidotaire du manuscrit lat. 7010 (milieu XIII ${ }^{\mathrm{e}} \mathrm{s}$.) ${ }^{24}$ se voit attribuer la même désignation que le lat. 7009, le premier folio porte la mention Liber antidotorum. Il présente 967 médicaments en 82 folios. L'ordre alphabétique, également souligné par des initiales ornées, se poursuit jusqu'au folio 77 (zegzenia minor). Ensuite la même main a copié des recettes d'autres médicaments sans classement particulier (jusqu'au $\mathrm{f}^{\mathrm{o}} 79 \mathrm{v}^{\circ}$ ), une liste de poids et mesures avec leurs symboles $\left(\mathrm{f}^{\mathrm{o}} 80-80 \mathrm{v}^{\mathrm{o}}\right)^{25}$, puis d'autres médicaments (jusqu'au $\left.\mathrm{f}^{\mathrm{o}} 81 \mathrm{v}^{\circ}\right)$. Le folio 82 comporte quant à lui des recettes de plusieurs mains postérieures. Il est à remarquer que ce manuscrit, contrairement au précédent, présente de nombreuses annotations et recettes ajoutées dans les marges par différents utilisateurs de cet ouvrage.

Le lat. $16187^{26}$ diffère en bien des points des témoins précédents. Le catalogue lui donne le titre de Antidotarium alphabeticum ${ }^{27}$. Il comporte 107

21. Basel, Universitätsbibliothek, Cod. Basiliensis D III 14, $\mathrm{f}^{\mathrm{o}}$ 9-134.

22. Il s'agit d'un manuscrit d'assez petit format $(23 \times 12,5 \mathrm{~cm})$, provenant de l'abbaye de Royaumont. Cf. A. Kramer, K. Scheidt, « Die Handschriften des Antidotarius magnus », loc. cit., p. $109, \mathrm{n}^{\circ} 4$.

23. Catalogus codicum manuscriptorum Bibliothecae Regiae, IV, op. cit., p. 303.

24. Ibid., p. 303. Le format est un peu plus grand $(25,3 \times 15,6)$. Il appartenait à la Bibliothèque des rois aragonais de Naples. $C f$. A. KRAMER, K. SCHEIDT, «Die Handschriften des Antidotarius magnus », loc. cit., p. 109, $\mathrm{n}^{\circ} 5$.

25. Inc. Pondera medicinalium signorum conati sumus narrare... D'après L. THORNDIKE, P. KiBRE, A catalogue of Incipits..., op. cit., col. 1058, il s'agit du Liber de ponderibus de Constantin.

26. $1^{\text {re }}$ moitié du XIII ${ }^{\mathrm{e}}$ siècle selon A. Kramer, K. ScheIDT, «Die Handschriften des Antidotarius magnus », loc. cit., p. $110, \mathrm{n}^{\circ} 6$; fin $\mathrm{XII}^{\mathrm{e}}$ selon J. BARBAUD, «Platearius et l'Antidotaire Nicolas", Actes du XXXII ${ }^{e}$ Congrès International d'Histoire de la Pharmacie, 312, 1996, p. 301-305, p. 304 ; fin du XII ${ }^{\mathrm{e}}$ s., provenant d'Allemagne de l'ouest ou du pays mosan d'après F. AvriL, C. Rabel, Manuscrits enluminés d'origine germanique, t. 1, X $X^{e}$-XIV ${ }^{e}$ s., Paris, 1995 , 108, p. 125-126; décrit dans La Médecine médiévale à travers les manuscrits de la Bibliothèque Nationale, op. cit., 61, p. 74-75. Ce manuscrit, comme le lat. 16191, fut légué au Collège de Sorbonne par l'un de ses membres, Jacques de Padoue, maître ès arts, médecine et théologie vers le milieu du XIV ${ }^{\mathrm{e}}$ siècle. Le legs semble avoir porté sur 22 manuscrits. $C f$. L. DelisLe, Le Cabinet des manuscrits de la Bibliothèque impériale, II, Paris, 1874, p. 156.

27. L. Deliste, Inventaire des manuscrits de la Sorbonne, Paris, 1870, p. 43. 
folios d'un format légèrement plus important $(27 \times 17 \mathrm{~cm})$ et présente 1239 remèdes. Le premier folio est manquant, le texte commence avec la fin de la recette de l'Antidotum Adrianum. C'est un manuscrit soigné, comportant des lettres ornées et historiées : un visage dans le D de Diapinidion et de Dauid regis potio ( $\mathrm{f}^{\circ} 19$ et 20), un personnage féminin pour le I de Igia $\left(\mathrm{f}^{\mathrm{o}} 41\right)$, un félin soufflant dans une corne pour le L de Litrotripon $\left(\mathrm{f}^{\mathrm{o}} 44 \mathrm{v}^{\circ}\right)$, le roi Mithridate sur un trône pour le Metridatum ( $\left.\mathrm{f}^{\mathrm{0}} 46\right)$, un autre félin dans le $\mathrm{Y}$ de Yreon ( $\mathrm{f}^{\mathrm{o}}$ 98). Les lettres majuscules sont alternativement rouges, ocre et bleues, des bouts de ligne rouges soulignent la fin de chaque paragraphe. De nombreux médicaments ne portent pas réellement de nom précis et sont qualifiés à partir de leurs propriétés : c'est le cas d'un grand nombre de préparations désignées par les termes antidotum, apozima, electuarium, emplastrum, par exemple ${ }^{28}$, qui sont distinguées par la précision très relative des maladies contre lesquelles elles sont prescrites. Nous trouvons ainsi un antidotum probatissimum ad capitis dolorem suivi quelques lignes plus loin d'un antidotum ad passionnes capitis. D'autres portent un nom précis lié à leur composant principal ou à leur origine : antidotum diacastoreum, pigra Galieni... L'ordre alphabétique est respecté jusqu'au folio $101 \mathrm{v}^{\mathrm{o}}$ avec la préparation à base de gingembre, zinziber conditum, puis suivent des recettes de médicaments copiées par d'autres mains. Au folio $106 v^{\circ}$ figurent les poids des différents composants employés.

Les témoins de l'Antidotarius magnus que nous venons d'évoquer présentent la particularité de ne contenir que le texte de l'Antidotaire et quelques annexes à l'exclusion de tout autre ouvrage important et d'être de présentation soignée, luxueuse même dans le cas du lat. 16187. Cependant, un autre antidotarium anonymi de la $\mathrm{BnF}$ semble également être apparenté à l'Antidotarius magnus. Le manuscrit lat. 15113 est présenté comme contenant des Traités de médecine parmi lesquels le Liber dinamidiorum Galieni et un Liber passionarius ${ }^{29}$. C'est un volume de 224 feuillets, de petit format, un recueil factice constitué de manuscrits d'époques différentes ${ }^{30}$ : une flebotomia et un Liber dinamidiorum Galieni copiés au XIV ${ }^{\mathrm{e}}$ siècle, l'ouvrage d'Isaac Israeli De diaetis particularibus copié au XIII ${ }^{\mathrm{e}}$ siècle, la partie centrale qui comporte l'antidotaire est la plus ancienne (fin $\mathrm{XII}^{\mathrm{e}}$ s.). Celui-ci commence au fo 167 avec la recette de l'Aurea alexandrina. L'écriture est de petite taille, assez peu soignée, seul le folio 167 comporte des initiales rouges ; ailleurs, l'empla-

28. Le terme d'antidotum, contrepoison en latin classique, a le sens de médicament au Moyen Âge. Apozima désigne une décoction dans de l'eau ou tout autre liquide ; les électuaires (confection de consistance molle obtenue à partir de poudres mélangées à du miel, un sirop ou une eau jusqu'à obtention de la consistance voulue) et emplâtres (préparations solides destinées à rester longtemps en place) apparaissent comme les formes médicamenteuses les plus représentées dans nombre d'antidotaires. Sur les différentes formes pharmaceutiques, voir J.-P. BÉNÉZET, Pharmacie et médicament..., op. cit., p. 545-635.

29. L. DelisLe, Inventaire des manuscrits de l'Abbaye de Saint-Victor, Paris, 1869, p. 73.

30. Cf. G. OuY, Les Manuscrits de l'abbaye de Saint-Victor, Turnhout, 1999, t. 2, p. 170 : ce recueil de $21 \times 13,5 \mathrm{~cm}$ rassemblait " 7 éléments sans doute réunis à la fin du $\mathrm{Xv}^{\mathrm{e}}$ siècle. Le premier ainsi que les folios de garde (notamment 224 et 225) ont disparu». 
cement de l'initiale est réservé mais est resté vide. Le classement par ordre alphabétique (assez approximatif parfois) se poursuit jusqu'au folio 196, avec la Yera pigra dont le texte est interrompu brutalement sans fournir la recette. Puis d'autres mains ont transcrit des recettes variées jusqu'au folio 198 .

Cet antidotaire, dont la nomenclature présente 301 médicaments, semble être une forme abrégée de l'Antidotarius magnus. C'est sans doute cette même volonté d'abréger ce volumineux ouvrage, peu maniable avec ses centaines de médicaments, qui est à l'origine de deux autres antidotaires dont la fortune fut bien différente, ainsi que le montre le nombre de manuscrits qui les contiennent respectivement : il s'agit du Liber iste attribué à Platearius et de l'Antidotarius Nicolai.

\section{Le Liber iste}

Cet ouvrage, dont l'attribution n'est pas encore certaine, est présenté par son auteur ${ }^{31}$ comme la compilation de plusieurs antidotaires : Liber iste qui in presentia nobis legendus occurrit ex multorum antidotis est compilatus ${ }^{32}$. Il comporte une sélection de médicaments mais aussi une série de commentaires et de réflexions plus théoriques ${ }^{33}$. Il a longtemps été considéré comme un commentaire à l' "Antidotaire de Nicolas », mais les travaux initiés par Alfons Lutz en $1960^{34}$ ont prouvé qu'il s'agissait bien plutôt d'un ouvrage découlant de l'Antidotarius magnus salernitain. Cependant, nombreux sont les manuscrits qui le présentent étroitement associé à l'Antidotarium Nicolai et c'est bien dissimulé, noyé même à l'intérieur du célèbre texte, qu'apparaît le Liber iste dans le manuscrit lat. $16191^{35}$. L'ouvrage intitulé Antidotarius

31. Il s'agit vraisemblablement de Matheus Platearius, auteur salernitain du milieu du XII ${ }^{\mathrm{e}}$ siècle. Cet ouvrage important, mais qui fut supplanté par l'Antidotaire de Nicolas, figure dans plus de trente manuscrits répertoriés à ce jour. $C f$. M. AusÉCACHE, "Un Liber iste, des Liber iste ? Un Platearius, des Platearius? État des lieux d'un projet d'édition », La Scuola Medica Salernitana. Gli autori e $i$ Testi, Actes du colloque de Salerne, 3-5 novembre 2004, à paraitre.

32. Ms. Reims, Bibliothèque municipale, 1002, fo 201.

33. Du moins dans la version «longue» de ce texte dont on peut lire deux états : une version A contenue dans le manuscrit Reims, Bibl. Mun. 1002 et une version B qui apparaît comme une amplification et un commentaire de la première et qui connut une plus grande fortune. Les développements de la seconde version portent surtout sur les vertus des différents simples utilisés, ces commentaires empruntant largement au Circa instans. Mais l'on peut également trouver des remarques plus théoriques concernant les mélanges et le mode d'action des médicaments. Autre différence entre les deux versions : le nombre de remèdes présenté est de 93 dans le témoin de Reims alors que dans les versions commentées ce nombre se situe entre 67 et 69. Les deux manuscrits de la BnF présentent la version B.

34. A. LUTZ, «Der verschollene frühsalernitanische Antidotarius magnus in einer Basler Handschrift aus dem 12. Jahrhundert und das Antidotarium Nicolai », Veröffent. der intern. Ges. für Gesch. der Pharmazie, NF, Bd. 16, 1960, p. 97-133. Voir également J. BARBAUD, op. cit.

35. L. DelisLe, Inventaire des manuscrits de la Sorbonne, op. cit., p. 45. Ce recueil datant du XIV ${ }^{\mathrm{e}}$ siècle a également appartenu à Jacques de Padoue qui en fit don à la Sorbonne. $C f$. M. GREEN, «The development of the Trotula », Revue d'Histoire des Textes, 26, 1996, p. 119203, sp. p. 167-168, 177. 
Nicolai cum glosulis, sans précision quant à l'auteur des gloses, est de fait le montage de deux textes. L'antidotarium Nicolai commence au folio $92 \mathrm{v}^{\circ}$ par l'incipit du prologue Ego Nicolaus... puis, au folio 93, nous trouvons la mention Liber iste quem in presenciarum legendum assumpsimus..., incipit du texte de Platearius. Les 69 médicaments du Liber iste sont insérés, sous forme de gloses, dans l'Antidotaire de Nicolas qui compte ici 137 préparations.

Une autre copie du Liber iste se trouve à la BnF. En effet le manuscrit lat. $6954^{36}$, également du XIV siècle, présente un Anonymi antidotarius commençant ainsi ( $\mathrm{f}^{\circ}$ 189) : Liber iste quem in presenciarum legendum assumpsimus ex multis antidotis est compilatus... et se terminant par la mention explicit liber iste ( $\left.\mathrm{f}^{\mathrm{o}} 228\right)$.

Le contexte manuscrit du Liber iste dans ces deux recueils est dominé par les auteurs salernitains : Musandinus, Ricardus et Salernus dans le lat. 6954, Ricardus et «Trotula» dans le lat. $16191^{37}$. Cependant, dans les deux cas, nous relevons également la présence d'auteurs considérés comme montpelliérains, Galterus Agilon et Rogerus de Barone ${ }^{38}$. Ces deux manuscrits ont la particularité de contenir à la fois l'Antidotarium Nicolai, le Liber iste et le Pomum ambre, mais ni l'un ni l'autre ne donnent les moyens d'identifier l'antidotaire attribué à Platearius.

\section{L'Antidotarium Nicolai}

Dernier représentant, et non le moindre, de la lignée salernitaine, cet antidotaire pose encore de multiples problèmes relatifs à ses date et lieu de composition, ou à son auteur ${ }^{39}$. Cet ouvrage qui connut une immense notoriété jusqu'à la fin du Moyen Âge figure dans de nombreux manuscrits et

36. Catalogus codicum manuscriptorum Bibliothecae Regiae, IV, op. cit., p. 296-297. Ce manuscrit in folio provient de l'hôpital de Beaune. Cf. L. DelisLe, Cabinet des manuscrits de la Bibliothèque Impériale, t. II, Paris, 1874, p. 339.

37. Musandinus, Ricardus et Salernus sont trois maîtres salernitains du XII ${ }^{\mathrm{e}}$ siècle, célèbres pour leurs ouvrages et la qualité de leur enseignement tant louée par Gilles de Corbeil ; $C f$. S. DE RENZI, Collectio salernitana, Naples, 1852-1857 ; Trotula désigne non pas un auteur mais un ensemble de traités consacrés aux «maladies des femmes », montage élaboré à Salerne à partir de la fin du $\mathrm{XI}^{\mathrm{e}}$ siècle. Cf. M. GreEn (éd., trad.), The Trotula. A Medieval Compendium of Women's Medicine, Philadelphie, 2001.

38. E. WiCKERSHEIMER, Dictionnaire biographique des médecins en France au Moyen Âge (1936), rééd. Genève, 1979, p. 170-173, 720-721; D. JACQUART, Supplément, Genève, 1979, p. 80-81, 263. Sur Montpellier voir L. Dulieu, La Médecine à Montpellier. T. 1 : le Moyen Âge, Avignon, 1975 ; D. LE BLEVEC (dir.), L’Université de médecine de Montpellier et son rayonnement $\left(X I I I^{e}-X V^{e}\right.$ s.), Actes du colloque international de Montpellier, Université Paul Valéry-Montpellier, 17-19 mai 2001, Turnhout, 2004.

39. Voir D. Goltz, Mittelalterliche Pharmazie und Medizin..., op. cit. J. Barbaud situe la rédaction de ce traité dans la région de Paris durant le premier tiers du XIII ${ }^{\mathrm{e}}$ siècle. $C f$. «Platearius et l'Antidotaire Nicolas », op. cit., p. 303-304. Cependant la filiation, au moins intellectuelle, avec Salerne est clairement établie par la nomenclature reprise presqu'entièrement de l'Antidotarius magnus. 
est en principe bien repérable, notamment grâce à son incipit Ego Nicolaus rogatus a quibusdam... Il n'y a donc pas, semble-t-il, de difficulté quant à l'identification de l'œuvre... Pourtant, nous constatons de notables différences dans les relevés établis par les différents auteurs ayant travaillé sur ce sujet: D. Goltz cite 7 manuscrits de la BnF contenant ce célèbre texte ${ }^{40}$, J. Barbaud en ajoute $9^{41}$, liste qu'il faut compléter par le lat. 6988A, le n.a.l. $1789^{42}$. La BnF est donc riche de 17 exemplaires de cet ouvrage, témoins présentant un nombre variable de médicaments, preuve du caractère « ouvert» et instable du texte ${ }^{43}$. Rappelons que l'édition princeps de 1471 qui figure en fac-similé dans l'ouvrage de D. Goltz comporte 142 préparations. Dans les manuscrits de la $\mathrm{BnF}$, nous relevons entre 113 et 163 recettes pour la plupart des témoins en plaçant à part les 2 manuscrits qui présentent le nombre le plus élevé de médicaments dont certains semblent d'origine incertaine. Le lat. 7058A, qui présente jusqu'à 423 médicaments, est un manuscrit tardif $\left(\mathrm{XV}^{\mathrm{e}} \mathrm{s}\right.$.) et se compose surtout de textes de pharmacopée et de synonyma ${ }^{44}$.

Le manuscrit n.a.1. 1789, également du $\mathrm{XV}^{\mathrm{e}}$ siècle, associe l'Antidotaire à deux ouvrages de Mesue et au De uenenis de Pietro d'Abano en italien. Ce témoin comporte 248 médicaments d'origines diverses. Aux médicaments « classiques » s'ajoutent par exemple des composés tels que le Barbatides quod facit ad omnes imbecillites et enfraxim stomachi ( $\left.\mathrm{f}^{\circ} 74 \mathrm{a}\right)$, le Diamacis de la Practica du Pantegni clairement citée (fo 80 a) ainsi que 1'Oleum Michaelis Scoti contra lumbricos ( $\mathrm{f}^{\mathrm{o}} 86 \mathrm{~b}$ ). Il faut également ajouter la présence d'un onguent Alabastrum nardi istici preciosi quod romany de Ierusalem attulerunt tempori quo dominus noster yhesus christus fuit crucifixus (fo 97b) ainsi que celle d'un Unguentum a quodam medico excellentissimo probatum fuit apud Montes Pessulanum $\left(\mathrm{f}^{\circ} 97 \mathrm{v}^{\circ} \mathrm{b}\right)$. L'Antidotaire de Nicolas apparaît dans ces manuscrits comme le noyau central autour duquel se concentrent des préparations plus récentes ou venues d'autres horizons.

Les autres témoins présentent une série de 114 à 163 médicaments, un texte donc beaucoup plus court et plus maniable que celui de l'Antidotarius magnus, concision qui explique sans doute en partie son succès. Les notices consacrées aux différents médicaments sont brèves, les prescriptions sont rapidement présentées, sans commentaires. Les dosages et modes d'administration sont donnés mais les opérations de confection des mélanges ne sont

40. Il s'agit des manuscrits lat. 6888, 6943A, 6954, 6988, 16176, 16191. Après examen, il s'avère que le manuscrit lat. 15362 cité par D. Goltz ne contient pas l'Antidotarium Nicolai.

41. Manuscrits lat. 6823, 6957, 7038, 7056, 7058A, 15116, n.a.1. 173, n.a.1. 343, n.a.1. 1485.

42. Deux témoins comportent des extraits de l'Antidotarium Nicolai : les lat. 11228, n.a.1. 693.

43. Les nombreux témoins du Circa instans présentent ce même caractère d'instabilité du texte qui peut contenir de 252 à 500 chapitres. C'est sans doute la matière même de ces œuvres, toujours sujettes à compléments ou amplification, puis leur succès qui les rend "flexibles », «adaptables ». Cf. I. VenturA, «Per una storia del Circa instans. I Secreta salernitana ed il testo del manoscritto London, British Library, Egerton 747 : note a margine di un'edizione », Schola Salernitana Annali, 7-8, 2002-2003, p. 39-109.

44. Il porte la mention (datée de 1609) ex libris Michaelis Lange philopharmaci parisiensis. 
pas précisées, ce qui peut indiquer que le public concerné par cet ouvrage connaît déjà les différents modes de préparation des remèdes ${ }^{45}$. Les ajouts postérieurs relevés dans bon nombre de manuscrits témoignent d'une utilisation réelle de ce texte considéré comme un ouvrage de base mais susceptible d'enrichissements ${ }^{46}$.

L'Antidotaire est souvent entouré d'ouvrages d'auteurs salernitains: Musandinus, Ricardus, Platearius (lat. 6954), «Trotula », Jean de Saint Paul, Constantin, Gilles de Corbeil (lat. 6988), mais il côtoie également des maitres montpelliérains : Galterus Agilon, Rogerus de Barone (lat. 6954, 7056, 16191), Bernard de Gordon (lat. 6957), Arnaud de Villeneuve (6988A). Dans deux témoins est relevée la présence d'ouvrages de Mesue ${ }^{47}$. Il faut remarquer que le n.a.l. 343 qui associe l'Antidotaire à une série d'œuvres de Galien est un recueil factice, l'ensemble Antidotaire, Alphita ${ }^{48}$, Synonyma étant un ajout en fin de volume. Nombre de manuscrits sont d'une facture soignée tels ces volumes qui comportent également le Viaticum ${ }^{49}$ commenté, en corrélation vraisemblablement avec l'enseignement universitaire parisien ${ }^{50}$. L'Antidotaire fut lui-même également l'objet de commentaires dont le plus célèbre est celui de Jean de Saint-Amand (fin XIII ${ }^{\mathrm{e}}$ s.), largement diffusé dans le cadre universitaire ${ }^{51}$. Dans une autre logique, le manuscrit lat. $6957\left(\mathrm{Xv}^{\mathrm{e}} \mathrm{s}\right.$.) associe ouvrages médicaux et traités d'astrologie ou de chiromancie, illustrant ainsi la variété des approches dont pouvait disposer le praticien dans l'exercice de

45. J.-P. BÉNÉZET, Pharmacie et médicament..., op. cit., p. 109: «L’Antidotarium Nicolai et les Opera du pseudo-Mésué (rédigés au XIII ${ }^{\mathrm{e}}$ s.) sont la base de la documentation professionnelle des apothicaires occidentaux ». Rôle durable puisque, toujours selon le même auteur, ce fut «L'importation des premières drogues d'Amérique du Sud [qui] imposa au XVII siècle un net remaniement de la littérature pharmacologique. Le bois de gaïac, l'ipéca et le quinquina, sonnèrent alors le glas de l'Antidotarium Nicolai. », ibid., p. 108.

46. Ainsi dans le lat. 7056, l'Antidotaire comporte 115 médicaments mais la même main a ajouté de nombreuses autres recettes dans les marges. Le lat. 6283 présente quant à lui une série de recettes intitulées Cetere confectiones et medicinis (sic) que non sunt in antidotario Nicholai.

47. Lat. $6943 \mathrm{~A}$ et n.a.1. 1789 .

48. Il s'agit d'une liste de synonyma commençant par alphita est farina ordei. Éditée par S. DE RENZI, Collectio salernitana, op. cit., III, p. 271-322.

49. Traduction par Constantin l'Africain de l'ouvrage de pathologie d'Ibn al-Gazzar, cet ouvrage fit très tôt partie des programmes de la Faculté de médecine de Paris. Il fut commenté par Girardus Bituricensis à la demande de ses collègues parisiens. $C f$. D. JACQUart, F. Micheau, La Médecine arabe..., op. cit., p.172-173.

50. Lat. 6888, 15116. Le lat. 16176 associe également l'Antidotaire à l'Isagoge, à Hippocrate et à Isaac Israeli, regroupement que nous retrouvons dans le lat. 7038 tandis que le n.a.1. 173 fait côtoyer Viaticum, Antidotaire de Nicolas, Isaac et Gilles de Corbeil. Ces associations font penser au programme établi par la faculté de médecine de Paris dans la deuxième moitié du XIII ${ }^{\mathrm{e}}$ siècle. $C f$. H. Denifle, E. Chatelain, Chartularium universitatis Parisiensis, t. I, Paris, 1889, p. 547.

51. D. JACQUART, «L'œuvre de Jean de Saint-Amand et les méthodes d'enseignement à la Faculté de médecine de Paris à la fin du XIII e siècle", dans J. HAMESSE (éd.), Manuels, programmes de cours et techniques d'enseignement dans les universités médiévales, Louvain-laNeuve, 1994, p. 257-275. 
ses fonctions ; l'Antidotaire fait alors partie d'un ensemble d'outils diversifiés. L'importance du nombre de témoins conservés et la diversité du contexte manuscrit montrent bien la grande diffusion et l'importance de ce texte à partir du XIII ${ }^{\mathrm{e}}$ siècle ${ }^{52}$. L'ouvrage dont il est question ensuite est loin d'avoir connu la même notoriété.

\section{Le Pomum ambre}

Cet antidotaire dont l'incipit est Pomum ambre duplicatum est ad reuma suspendendum et contra debilitatem cerebri figure souvent dans les catalogues sous l'appellation d'anonymi antidotarium. Il se trouve dans 6 manuscrits de la $\mathrm{BnF}^{53}$. Paul Meyer le présente ainsi : « une compilation de remèdes dont on possède divers manuscrits et qui a été traduite en français et en anglais. Les remèdes sont classés selon la forme sous laquelle ils se présentent: poudres, électuaires, pilules, sirops, emplâtres, onguents ${ }^{54}$. On trouve effectivement ces 6 chapitres dans les différents manuscrits consultés, chaque médicament y est rapidement présenté, l'essentiel étant la recette ${ }^{55}$. Cet ouvrage semble donc rompre doublement avec la tradition établie par Salerne : classement des médicaments par ordre alphabétique, nom précis élaboré en fonction du principal composant ou du nom de l'inventeur présumé. Dans le Pomum ambre, au contraire, ces désignations précises sont rares et le plus souvent le remède est simplement désigné par une ou plusieurs mentions de prescription: puluis contra lithiasim, puluis contra quartanam et uicium splenis, sirupus ualens macilentis colericis et extenuatis... Les désignations plus précises existent cependant, rappelant parfois celles de l'Antidotarius magnus : electuarium dyanisum, electuarium athanasii, stomatichon, pilule arnoglosse, par exemple. Mais d'autres appellations semblent bien provenir d'autres sources : electuarium quod manus dei uocatur, sirupus contra malum mortuum et le même unguentum alabaustri nardi pistici preciosi déjà présent dans la version tardive de l'Antidotarium Nicolai du n.a.l. $1789^{56}$.

52. Rappelons qu'il fut traduit en langue vernaculaire. $C f$. P. Dorveaux, L'Antidotaire Nicolas..., op. cit.; W. Braekman, G. KeIL, «Fünf mittelniederländische Übersetzungen des Antidotarium Nicolai », Sudhoffs Archiv, 55.3, 1971, p. 257-320.

53. Les lat. 6887A, 6954, 6957, 7056, 14026, 16191.

54. P. MeYer, «Les manuscrits français de Cambridge», Romania, 32, 1903, p. 268. Le Pomum ambre est présenté par L. ThORNDIKE et P. KIBRE comme un antidotarius sans autre précision. Cf. A catalogue of Incipits, op. cit., 1057. Outre les six témoins de la BnF, une vingtaine d'autres manuscrits ont été recensés à ce jour.

55. Le texte choisi comme référence pour l'étude de cet ouvrage est celui du manuscrit BnF lat. $16191, \mathrm{f}^{\circ} 193 \mathrm{v}^{\circ} \mathrm{b}-201 \mathrm{v}^{\circ} \mathrm{b}$. Dans cette copie nous pouvons dénombrer 41 recettes de poudres, 24 électuaires, 34 pilules, 60 sirops, 41 emplâtres, 44 onguents.

56. Outre la mention faisant référence à la crucifixion, les prescriptions s'accompagnent d'un commentaire citant les deux figures bibliques de Marthe et Marie : «Unguentum alabaustri nardi pistici preciosi quod romani de Ierusalem attulerunt tempore quo dominus noster Ihesus Christus cruxifixus fuit, probatum omnibus febribus et frigoribus, calculosis, lumborum doloribus. Petram de uesica rumpit, dolorem aufert et emigraneum dolorem amputat, cause matricis 
Autre originalité de cet antidotaire, des recettes de composés en rapport avec la cuisine, telles ces puluis ad salsamenta et piperatas, puluis ad galentinam et salsamenta specialia, recettes destinées à faciliter la digestion ${ }^{57}$. D'autres médicaments paraissent liés à une pratique chirurgicale précise, ainsi cet onguent appliqué avec du vin soufré et de l'huile après une intervention clairement décrite :

... post sedationem tumoris elargetur uulnus et apponatur terebentina cum euforbium. Si autem delicatus sit et non patiatur maiorem elargationem uulneris apponatur hoc post unguento et emplastro ${ }^{58} \ldots$

Le public visé par cet antidotaire semble donc être moins le cercle des médecins universitaires que le vaste monde des praticiens, barbiers et apothicaires, tant ce texte paraît éloigné de la médecine savante et des réflexions théoriques qui en sont la marque. Cependant, des noms de maîtres connus ou d'autorités apparaissent parfois, ce qui complique encore la question des sources de cet ouvrage. Ainsi, dans le lat. 16191 trouvons-nous les noms de Nicolas (electuarium ad restaurationem humiditatis secundum magistrum nicholaum), Maurus (2 recettes de pilules), Constantin (citation du Viaticum), Iohannes de Sancto Egidio ${ }^{59}$, Stephanus Salernitanus ${ }^{60}$. Il faut noter des variantes quant aux noms cités dans les différents manuscrits considérés : le lat. 7056 présente une Puluis magistri Iohannis Lombardi, le lat. 6887A attribue à Diacorides la recette dont le lat. 16191 donnait la paternité à Stephanus salernitanus, les manuscrits lat. 14026 et lat. 6954 mentionnent à la place de Iohannes de Sancto Egidio un Robertus de Sancto Egidio dont nous n'avons pas trouvé trace. C'est l'aspect pratique de l'enseignement des maîtres qui

optime subuenit. Maria et Martha use fuerunt hoc unguento omnibus diebus uite sue » (lat. $\left.16191, \mathrm{f}^{\mathrm{o}} 200 \mathrm{v}^{\mathrm{o}} \mathrm{b}\right)$.

57. D'après Bruno Laurioux, que je remercie pour cette communication personnelle, les poudres pour faire gelées et sauces sont parmi les rares recettes de cuisine à figurer hors des recueils proprement culinaires. On est à la limite de la cuisine et de la médecine, c'est la présence de mesures de proportion dans les recettes d'ordre médical qui fait la différence.

58. Lat. $16191, \mathrm{f}^{\mathrm{o}} 199 \mathrm{v}^{\circ} \mathrm{b}$.

59. Cf. E. WicKERSHEIMER, Dictionnaire biographique des médecins en France, Genève, 1979, t. II, p. 478-79 ; D. JACQUarT, Supplément, op. cit., p. 180. D'origine anglaise, il étudia sans doute les arts libéraux et la médecine à Oxford, Paris et Montpellier, puis enseigna la médecine dans l'une ou l'autre de ces deux dernières villes. Il retourna en Angleterre en 1235 et devint archidiacre d'Oxford en 1240. En 1253, il fut appelé comme "médecin du corps et médecin de l'âme» au chevet de Robert Grosseteste mourant et en 1258 il guérit du poison le comte de Gloucester. Il ne reste de ses œuvres médicales que des Experimenta et des recettes portant son nom. Il serait mort vers 1259 .

60. Ce personnage semble assez difficile à identifier. Dans la liste des salernitains dressée par S. de Renzi figure un seul Stephanus: Stefano Mondezario (fin XIII ${ }^{\mathrm{e}} \mathrm{s}$ ), médecin réputé semble-t-il, puisqu'il fut appelé à la cour de Constantinople pour soigner le fils de l'empereur. Cf. S. DE RenZI, Collectio salernitana, op. cit., I, p. 335. Il existe également un Iohannes Stephanus, présent dans certains manuscrits avec ses Curae ou son court traité De dosibus. E. WicKERSHEIMER, Dictionnaire biographique..., op. cit. D. JACQUART, Suppl., op. cit., p. 184, situe Iohannes Stephanus à Montpellier. 
est retenu, leur nom étant associé à des recettes; les utilisateurs de l'antidotaire semblent être eux-mêmes des praticiens expérimentés puisqu'à la fin de l'ouvrage on trouve la mention suivante :

Notandum quod hic est modus generalis faciendi unguenta de quibuscumque herbis uolueris quas uolueris ad tuam causam ualere...

Le Pomum ambre est un antidotaire original par rapport aux précédents, original dans sa composition et sans doute dans ses sources. La question de son origine et de sa date de composition est complexe. Il est selon toute vraisemblance plus tardif que les précédents puisqu'il mentionne Iohannes de Sancto Egidio actif dans la première moitié $\mathrm{du} \mathrm{XIII}^{\mathrm{e}}$ siècle. Les manuscrits les plus anciens du Pomum ambre conservés à la BnF datent de la fin du $\mathrm{XIII}^{\mathrm{e}}$ siècle et le contexte manuscrit fournit quelques éléments de réflexion. Dans le lat. 6887A, l'antidotaire est associé à la Practica (incomplète) du Pantegni mais il s'agit de deux unités codicologiques différentes, le Pomum ambre « compensant » peut-être l'absence de l'antidotaire de Constantin. Dans les cinq autres manuscrits recensés (lat. 6954, 6957, 7056, 14026, 16191), nous pouvons distinguer deux groupes de textes et d'auteurs: un groupe « salernitain » représenté par Constantin, Musandinus, Platearius, Salernus, Ricardus..., et un ensemble qui s'apparente davantage à Montpellier avec Galterus Agilon, Rogerus de Barone, Pontius de Sancto Egidio... Cette observation est sans doute à rapprocher du fait que les médicaments présentés dans cet antidotaire semblent provenir de diverses origines. Une dimension « française », sans doute méridionale, est indiquée par les manuscrits lat. 6954, 7056, 14026, 16191 qui présentent une puluis patriarche qui gallice dracheta uocatur $^{61}$. Le manuscrit 6887A, quant à lui, présente un ajout à l'intérieur de la recette d'un electuarium ad lumbricos: il est précisé qu'il serait plus actif cum herba que uocatur waldeia, ce dernier terme étant inscrit par un copiste différent dans un emplacement réservé ${ }^{62}$.

Cet ouvrage nous semble donc présenter l'intérêt d'être une sorte de synthèse de diverses influences; surtout il paraît répondre à une logique et à une finalité assez différentes des antidotaires précédents. Il peut être considéré comme étant en priorité destiné à ceux qui préparent les médicaments en fonction des prescriptions du médecin. La partie théorique et explicative a complètement disparu, laissant la place aux seules recettes classées par type de médicament, classement plus commode pour un exécutant, marquant ainsi la distance entre le médecin et l'apothicaire.

61. Drageta dans le manuscrit lat. 7056.

62. Il s'agit peut-être de la waldrian (valériane) répertoriée par W. F. DAEMS, Nomina simplicium medicinarum ex synonymariis medii aevi collecta, Leyde-New York-Cologne, 1993, p. 62. Ce terme se trouve au folio 38 du manuscrit de Vienne, Österreichische NationalBibliothek, ms. 5295, qui contient un Vocabularius latino-germanicus de nominibus plantarum ordine alphabetico constitutus (fo 38-42). 
L'évolution des antidotaires apparaît bien comme un reflet de l'évolution de la pensée médicale médiévale et de la pratique thérapeutique. La figure du médecin préparant lui-même les remèdes selon des recettes classées par type de maladie est présente jusqu'au XII ${ }^{\mathrm{e}}$ siècle, époque à laquelle une nouvelle orientation se fait jour, sous l'influence, entre autres, de Salerne ${ }^{63}$. Le classement alphabétique des médicaments s'impose et surtout des ouvrages comme le De gradibus de Constantin ou le Circa instans de Platearius permettent de satisfaire le besoin d'explication, de théorisation particulièrement visible dans le Liber iste. Cependant, la spécialisation croissante des professions de santé aboutit à la conception d'antidotaires plus simples, plus faciles d'utilisation pour les apothicaires, les théories médicales étant développées dans d'autres types de textes. Dans le même temps, ces antidotaires s'enrichissent de nouveaux médicaments issus d'autres traditions, d'autres auteurs, d'autres besoins ${ }^{64}$. La riche tradition manuscrite du Pomum ambre et surtout de l'Antidotarium Nicolai atteste le succès de ces ouvrages « ouverts » et le fonds de la $\mathrm{BnF}$ est représentatif de l'inégale fortune des antidotaires médiévaux.

Mireille AusÉcache, EPHE, IV ${ }^{\mathrm{e}}$ section, 45-47, rue des Écoles, F-75005 Paris.

\section{Manuscrits d'antidotaires médiévaux : quelques exemples du fonds latin de la Bibliothèque nationale de France}

Les antidotaires, recueils de médicaments composés, sont de précieux témoins de l'évolution de la médecine médiévale, évolution marquée par le tournant des $\mathrm{XI}^{\mathrm{e}}-\mathrm{XII}{ }^{\mathrm{e}}$ siècles, sous l'influence de Salerne. Généralement inédits, leur étude passe par l'examen des témoins manuscrits qui les conservent, tâche souvent rendue difficile par l'imprécision de la plupart des catalogues de bibliothèques

63. De même, la figure du moine préparateur et thérapeuthe, si elle ne disparaît pas, s'efface sous l'effet d'une laïcisation et d'une professionnalisation de la médecine. $C f$. E. WiCKERSHEIMER, «L'évolution de la profession médicale au cours du Moyen Âge », Scalpel, 1924, p. 675-715.

64. La recrudescence des cas de lèpre dès le début du XII ${ }^{\mathrm{e}}$ siècle amène les médecins occidentaux à compléter la pharmacopée héritée de l'Antiquité et à préparer de nouveaux composés à partir de simples locaux ou d'épices importées telles que poivre, cannelle, noix de muscade, gingembre. $C f$. J.-N. BIRABEN, «Les maladies en Europe : équilibres et ruptures de la pathocénose », dans M. D. GrmeK (dir.), Histoire de la pensée médicale en Occident. T. 1 Antiquité et Moyen Âge, Paris, 1995, p. 283-310, sp. p. 307 ; F. O. Touatı, Maladie et société au Moyen Âge. La lèpre, les lépreux et les léproseries dans la province ecclésiastique de Sens jusqu'au milieu du XIV siècle, Paris-Bruxelles, 1998, p. 163-164. L'épidémie de peste provoque à son tour le même phénomène, transformant, par exemple, la recette du Pomum ambre: ce médicament d'abord préconisé ad reuma suspendendum et contra debilitatem cerebri contient alors une faible quantité d'ambre ( 5 scrupules soit un peu plus de 6 grammes); la formule destinée aux malades de la peste comporte quant à elle 3 drachmes d'ambre (plus de 11 grammes). Le manuscrit lat. 16191 de la BnF comporte les deux recettes du Pomum ambre, la première formule est celle qui donne son titre à l'antidotaire $\left(\mathrm{f}^{\circ} 193 \mathrm{v}^{\circ} \mathrm{b}\right)$, la recette «enrichie » se trouve placée à la fin, à la suite de la série des onguents ( ( $\left.{ }^{\circ} 201 v^{\circ} \mathrm{a}\right)$. Le manuscrit lat. 6941 présente des Recepta et consilia $\left(\mathrm{f}^{\circ} 63 \mathrm{~b}-65 \mathrm{v}^{\circ} \mathrm{a}\right)$ commençant par une Descriptio pomi manutenendi contra pestem secundum Marsilium (merci à L. Moulinier-Brogi de m'avoir signalé ce manuscrit). 
et le faible nombre de répertoires spécialisés. Le fonds de la Bibliothèque nationale de France est riche en manuscrits médicaux contenant des antidotaires. Le type de recueil dans lequel ils figurent, les éventuelles traces d'utilisateurs ou de possesseurs sont autant d'indications sur la place occupée par ces textes dans la pratique médicale au Moyen Âge. L'étude porte principalement sur quatre antidotaires, trois ouvrages portant la marque de Salerne : l'Antidotarius magnus, le Liber iste attribué à Platearius, l'Antidotarius Nicolai et un recueil très différent des précédents, le Pomum ambre.

Antidotaires médiévaux - Salerne - Manuscrits médicaux - Antidotarius magnus - Liber iste - Antidotarium Nicolai - Pomum ambre

\section{Medieval Antidotaries Manuscripts : Some Examples from the «Bibliothèque nationale de France » Latin Fund}

Antidotaries, collections of compound medicines, are precious witnesses of medieval medicine evolution which is marked by the 11-12th turning-point which takes place under the influence of Salerno. Mostly unpublished, they must be studied by the way of their manuscripts, the difficulty coming from the imprecision of libraries catalogues and the small number of specialized indexes. The «Bibliothèque nationale de France » fund is rich in medical manuscripts inclosing antidotaries. The kind of collection where we find them, the possible readers or owners'marks are indications about their place in medical practice during the Middle Ages. Four antidotaries will be studied, three marked by Salerno: the Antidotarius magnus, the Liber iste ascribed to Platearius, the Antidotarius Nicolai and a very different one, the Pomum ambre.

Medieval antidotaries - Salerno - Medical manuscripts - Antidotarius magnus - Liber iste - Antidotarium Nicolai - Pomum ambre 


\section{Annexe : liste des manuscrits}

Cette présentation rapide est simplement destinée à cerner le contexte dans lequel se trouvent les œuvres considérées.

lat. $6823, \mathrm{XIV}^{\mathrm{e}} \mathrm{s}$.

Manfredus de Monte Imperiali, Liber de herbis et plantis; De avibus et piscibus ; Nicolai antidotarius $\left(\mathrm{f}^{\mathrm{o}} 185 \mathrm{a}-200 \mathrm{v}^{\circ} \mathrm{a}\right)$; Doses ; Quid pro quo ; Constantinus Africanus, Pondera medicinalia; Cetere confectiones et medicinis (sic) que non sunt in antidotario Nicholai; Simo Ianuensis, Sinonima abreviata per magistrum Mundinum

lat. $6887 \mathrm{~A}, \mathrm{XIII}^{\mathrm{e}} \mathrm{s}$.

Pantegni Practica (inc.); Pomum ambre (fo 119a-123b)

lat. $6888, \mathrm{XIV}^{\mathrm{e}} \mathrm{s}$.

Constantinus, Viaticum cum comm. Girardi Bituricensis; Iohannes de Sancto Amando, Glose super antidotarium; Antidotarium Nicholai (f $\left.\mathrm{f}^{\circ} 148 \mathrm{a}-156 \mathrm{v}^{\circ} \mathrm{a}\right)$; Doses ; Synonyma

lat. 6943A, XIV ${ }^{\mathrm{e}}$ s.

Mesue, Liber de consolatione medicinarum simplicium solutivarum; Mesue, Grabadim; Synonyma; Nicholai antidotarius ( $\mathrm{f}^{\circ}$ 63a-72a); Pondera ; Quid pro quo

lat. 6954, $\mathrm{XIV}^{\mathrm{e}} \mathrm{s}$.

Galterus Agilon, De urinis; Rogerus de Barone, Practica; Rogerus de Barone, Rogerina minor; Petrus Musandinus, Summula de preparatione cibium et potuum infirmorum; Ricardus Anglicus, De signis et pronosticis; Antidotarium Nicolai (fo $\left.103-115 v^{0} a\right)$; Platearius, Circa instans ; Iohannes Platearius, Practica ; Platearius, Liber iste super antidotarium $\left(\mathrm{f}^{\circ} 189 \mathrm{a}-228 \mathrm{v}^{\circ} \mathrm{a}\right)$; Pomum ambre $\left(\mathrm{f}^{\circ} 228 \mathrm{v}^{\circ} \mathrm{b}-\right.$ $\left.240 \mathrm{v}^{\circ}\right)$; Iohannes de Sancto Amando, Expositio super antidotarium Nicolai; Salernus, Tabulae

lat. $6957, \mathrm{XV}^{\mathrm{e}} \mathrm{s}$.

Alphita ; Petrus Hispanus, Tractatus mirabilis aquarum; De oculis tenebrosis ; Constantinus Africanus, De regimine sanitatis (Pantegni Practica, extr.) ; Bernardus Pictaviensis, De pulsibus; Ricardus Anglicus, Micrologus; De sterilitate; Bernardus de Gordonio, Libellus de impregnatione; Ps.-Arnaldus de Villanova, De sterilitate ; Iohannes Iacobi, Secretarium practice medicine; De confectionibus; Galterus Agilon, Dosis medicinarum; De oleis ; [Romualdus Salernitanus], Tractatus de morbis uniuersalibus et eorum nomina; Virtutes aliquarum herbarum ; Consilia medica uaria ; Iohannes Iacobi, De pestilentia ; Iohannes de Tornamira, De pestilentia; Guido de Caulhiaco, De gradibus medicinarum; De flebotomia; Regule de urinis ; Chiromancia ; Versus de complexionibus ; Astrologia; Tractatus de signis, Zodiacus est circulus oblicus in 12 partes...; De proprietatibus planetarum; De diebus septimane; Versus medicinales; Recepte; Antidotarium Nicolai (fo $168-184 v^{\circ}$ ) ; Recepte; Tractatus de sirupis ; Varia ad practicam; De sirupis; Tractatus de urinis optimus secundum Avicennam; Pomum ambre $\left(\mathrm{f}^{\mathrm{o}} 205 \mathrm{v}^{\mathrm{o}}-215 \mathrm{v}^{\circ}\right)$ 
lat. 6988, début XIV's.

Antidotarius Nicolai $\left(\mathrm{f}^{\mathrm{o}} 4 \mathrm{a}-21 \mathrm{v}^{\mathrm{o}} \mathrm{a}\right)$; Doses ; Additiones ad Antidotarium Nicolai ; Ps.-Avicenna, Flebotomia ; Constantinus, Liber stomachi; Constantinus, Liber de elephantia ; Pontius de Sancto Egidio, Cure ; Salernus, Compendium ; Constantinus, De coitu; Iohannes de sancto Paulo, Tractatus de medicinarum simplicium virtutibus ; [Petrus Hispanus ?], De phlebotomia ; De ordinatione diete secundum Constantinum ; Nicolaus, Quid pro quo ; Ricardus Anglicus, Anatomia ; [Zacharias], Tractatus de passionibus oculorum; Iohannes Stephanus, De febribus ; Trotula; Aegidius Corboliensis, De urinis cum commento Gilberti ; Aegidius Corboliensis, De pulsibus cum commento; Ps.-Galenus, Liber de spermate; Constantinus, Liber de melancolia

lat. $6988 \mathrm{~A}, \mathrm{XV}^{\mathrm{e}} \mathrm{s}$.

Trotula; Antidotarius Nicolai $\left(\mathrm{f}^{\mathrm{o}} 17-47 \mathrm{v}^{\circ}\right)$; De pondere herbarum; Alphita; Recepte; Arnaldus de Villanova, Tabula syruporum, clisteriorum et suppositorum; Recepte et notata pro impregnatione mulierum; Aegidius Cancellarius, De signis et curatione fistule; Astrologia; Aegidius Cancellarius, Experimenta; Secreta Ypocratis; Aegidius Cancellarius, Signa distinctiva de urinis; De herbis ex Isidoro ; Arnaldus de Villanova, Cure breves; Contra impedimentum conceptionis; Recepte

lat. 7009 , XIII ${ }^{\mathrm{e}}$ s.

Antidotarius magnus

lat. $7010, \mathrm{XIII}^{\mathrm{e}} \mathrm{s}$.

\section{Antidotarius magnus}

lat. $7038, \mathrm{XIV}^{\mathrm{e}} \mathrm{s}$.

Isaac Israeli, De diaetis particularibus ; Constantinus Africanus, Viaticum ; Iohannitius, Isagoge ; Hippocrates, Aphorismi (extr.) ; Antidotarium Nicolai ( $\mathrm{f}^{\mathrm{o}}$ 71$\left.76 \mathrm{v}^{\circ}\right)$; [Archimattheus], Modus medendi

lat. 7056, XIII ${ }^{\mathrm{e}} \mathrm{s}$.

Ricardus Anglicus, Anathomia; Ricardus Anglicus, De diebus criticis ; Ricardus Anglicus, Practica ; Rogerus de Barone, Practica sive Rogerina maior; Rogerus de Barone, Rogerina minor ; Trotula ; Trotula minor, de ornatu faciei ; Cleopatra, Gynaecia [abbreviata]; Non omnes quidem; Gilbertus Anglicus, Experimenta; Trotula, Tractatus de egritudinibus mulierum; Pomum ambre (fo 100a-108b); De ponderibus ; Alphita; Synonima antidotarii Nicolai; Galenus, Antibalomenon; Antidotarium Nicolai (fo 123a-147a) ; Versus de ponderibus medicinarum

lat. $7058 \mathrm{~A}, \mathrm{XV}^{\mathrm{e}} \mathrm{s}$.

Antidotarium Nicolai $\left(\mathrm{f}^{\mathrm{o}} 1 \mathrm{a}-152 \mathrm{v}^{\mathrm{o}} \mathrm{b}\right)$; Nicolaus, Doses ; Otho Cremonensis, Versus de simplicibus aromaticis; Quid pro quo; De confectionibus ex antidotario Nicolai; Alphita; Synonyma; Recepte

lat. 14026, XIII ${ }^{\mathrm{e}}$ s. (désordre des cahiers et de certains folios)

Pontius de Sancto Egidio, Summa; Pomum ambre $\left(1^{\text {re }}\right.$ partie, $\left.f^{\mathrm{o}} 52-60 \mathrm{v}^{\mathrm{o}}\right)$; Rogerus de Barone, Rogerina minor (extr.) ; Pomum ambre ( $3^{\mathrm{e}}$ partie, $\left.\mathrm{f}^{\mathrm{o}} 69-78\right)$; Galterus 
Agilon, Dosis medicinarum ( $2^{\mathrm{e}}$ partie) ; Ricardus Anglicus, De repressivis ; Galterus Agilon, Dosis medicinarum ( $\mathrm{r}^{\mathrm{re}}$ partie); Pomum ambre $\left(4^{\mathrm{e}}\right.$ partie, $\left.\mathrm{f}^{\mathrm{o}} 91\right)$; Pomum ambre $\left(2^{\mathrm{e}}\right.$ partie, fo 92$)$

lat. $15113, \mathrm{XIII}^{\mathrm{e}} \mathrm{s}$.

Flebotomia ; Galenus, De dinamidiis ; [Constantinus], Viaticum ? (extr.) ; Galenus, Liber passionarius ; Antidotarius magnus? (fo 167-196); Isaac Israeli, De diaetis particularibus

lat. $15116, \mathrm{XIV}^{\mathrm{e}} \mathrm{s}$.

Constantinus Africanus, Viaticum cum Giraudina ; Antidotarium Nicholai (f ${ }^{\circ}$ 135a151b) ; Ricardus Anglicus, Regule de urinis (extr.)

lat. $16176, \mathrm{XIII}^{\mathrm{e}} \mathrm{s}$.

Iohannitius, Isagoge ; Hippocrates, Aphorismi ; Hippocrates, Prognostica ; Hippocrates, Liber de regimine acutorum; Isaac Israeli, De urinis; Isaac Israeli, De febribus; Isaac Israeli, Diete universales; Isaac Israeli, Diete particulares; Constantinus Africanus, Viaticum ; Antidotarius Nicolai (fo 265a-284a)

lat. 16187, XIII ${ }^{\mathrm{e}} \mathrm{s}$.

Antidotarius magnus

lat. $16191, \mathrm{XIV}^{\mathrm{e}} \mathrm{s}$.

Rogerus de Barone, Practica; Rogerus de Barone, Parva summa; Platearius, Circa instans ; Antidotarius Nicolai $\left(\mathrm{f}^{\circ} 92 \mathrm{v}^{\circ}-151 \mathrm{v}^{\circ}\right)$; Platearius, Liber iste $\left(\mathrm{f}^{\circ}\right.$ 93$\left.151 v^{\circ}\right)$; Gerardus Cremonensis, Summa de modo medendi; Ricardus Anglicus, De signis pronosticis; Pomum ambre $\left(\mathrm{f}^{\mathrm{o}} 193 \mathrm{v}^{\circ} \mathrm{b}-201 \mathrm{v}^{\circ}\right)$; Galterus Agilon, De dosis medicinarum ; Trotula, de passionibus mulierum ; Alphita ; Sinonima; Quid pro quo

n.a.1. $173, \mathrm{XIII}^{\mathrm{e}} \mathrm{s}$.

Constantinus Africanus, Viaticum ; Isaac Israeli, Liber febrium ; Aegidius Corboliensis, De urinis ; Antidotarium Nicolai (fo $250 \mathrm{a}-286 \mathrm{v}^{\circ}$ )

n.a.1. $343, \mathrm{XIV}^{\mathrm{e}} \mathrm{s}$.

Galenus, De elementis secundum Hippocratem; Galenus, De complexionibus; Galenus, De malitia complexionis diverse ; Recepte; Ps.-Galenus, De virtutibus nostrum corpus dispensantibus ; Galenus, De somniis ; Galenus, Ad Glauconem de medendi methodo ; Galenus, De vinis ; Galenus, De voce et hanelitu; Galenus, De cibis ; Liber Nicholay (f $\left.{ }^{\circ} 130 \mathrm{a}-146 v^{\circ} a\right)$; Alphita ; Synonyma Nicholay

n.a.1. $1485, \mathrm{XIV}^{\mathrm{e}}$ s.

Constantinus, Pantegni theorica; Iohannes de Sancto Amando, Super antidotarium Nicolai; Nicolaus, Antidotarium (inc., fo 129a-139a); Iohannes Stephanus, De dosibus medicinarum (inc.)

n.a.1. $1789, \mathrm{Xv}^{\mathrm{e}} \mathrm{s}$.

Mesue, De consolatione medicinarum; Mesue, Grabadin; Recepte; Nicolai Antidotarium (fo $71 \mathrm{a}-98 \mathrm{v}^{\circ} \mathrm{b}$ ) ; Petrus de Apono, De uenenis (en italien) 
